\title{
Questes
}

\section{Hôpitaux de fondation comtale et pèlerins à Lille à la fin du Moyen Âge}

Irène Strobbe

\section{(2) OpenEdition}

1 Journals

\section{Édition électronique}

URL : http://journals.openedition.org/questes/1435

DOI : 10.4000/questes. 1435

ISSN : 2109-9472

Éditeur

Les Amis de Questes

\section{Édition imprimée}

Date de publication : 15 novembre 2011

Pagination : 50-64

ISSN : 2102-7188

\section{Référence électronique}

Irène Strobbe, "Hôpitaux de fondation comtale et pèlerins à Lille à la fin du Moyen Âge », Questes [En ligne], 22 | 2011, mis en ligne le 01 janvier 2014, consulté le 19 avril 2019. URL : http:// journals.openedition.org/questes/1435; DOI : 10.4000/questes.1435 


\title{
Hôpitaux de fondation comtale et pèlerins à Lille à la fin du Moyen Âge
}

\author{
Irène STROBBE
}

Depuis les années 1970, pour aider à la reconstitution des chemins de Saint-Jacques, les études de géographie des chemins de pèlerinage ${ }^{1}$ sont croisées avec celles de géographie hospitalière ${ }^{2}$. Historiens, archivistes et passionnés, souvent unis par l'expérience personnelle et intense de la marche au long cours vers Compostelle, mènent ces recherches au sein d'associations comme celle des Amis de Saint-Jacques ${ }^{3}$. La fatigue des corps, les moments de découragement devant le chemin qui reste à parcourir et le désir de trouver, à la fin d'une journée parfois éprouvante, un toit accueillant pour reprendre des forces ont pu contribuer à placer les archives hospitalières au cœur des études sur les pèlerinages. Les fonds de ces maisons charitables, a priori créées pour accueillir malades et pèlerins, ont été utilisés afin de brosser le portrait du pieux voyageur. Relier par des droites et des courbes les points formés par les implantations hospitalières pour tracer la carte des chemins de pèlerinage est une démarche ancienne et régulièrement reprise ${ }^{4}$. Mais savoir qui, de la route ou de l'hôpital,

\footnotetext{
${ }^{1}$ Voir en particulier Henri Treuille, «Autour d'une variante du chemin de Saint-Jacques à Toulouse vers le haut Comminges » et René de LA Coste-Messeliere et Gérard JugnOT «L'accueil des pèlerins à Toulouse », in Le Pèlerinage, Toulouse, Privat, "Cahiers de Fanjeaux », 15, partie « Géographie », 1980, respectivement p. 99-116 et p. 117-135.

${ }^{2}$ Voir la troisième partie du treizième colloque de Fanjeaux, «Éléments de géographie de l'hospitalité », in Assistance et charité, Toulouse, Privat, "Cahiers de Fanjeaux », 13, 1978 ; voir plus récemment Hôpitaux et maladreries au Moyen Âge : espace et environnement. Actes du colloque international d'Amiens-Beauvais (novembre 2002), Pascal MonTAUBIN (dir.), Histoire et archéologie médiévale, 17 (2004) et Les Établissements hospitaliers en France du Moyen Âge au XIX ${ }^{\mathrm{e}}$ siècle. Espaces, objets et populations, Sylvie LE CLECH-CHARTON (dir.), Dijon, Éditions universitaires de Dijon, « Sociétés », 2010.

${ }^{3}$ C'est au sein de cette association que René de La Coste-Messelière a fondé le Centre d'études, de recherche et d'histoire compostellanes.

${ }^{4}$ Cf. Marie-Claude Guigue, Les Voies antiques du Lyonnais, du Forez, du Beaujolais, de la Bresse, de la Dombes, du Bugey et de partie du Dauphiné déterminées par les hôpitaux du Moyen Âge, Lyon, H. Georg, 1877 ; Françoise BERIAC, «Hôpitaux et routes des Landes $\mathrm{XIII}^{\mathrm{e}}-\mathrm{XVI}^{\mathrm{e}}$ siècles », in André KLINGEBIEL et Jean-Bernard MARQUETTE (dir.), La Grande
} 
était là le premier, voilà une question à laquelle les historiens ont tenté de répondre sans toutefois y parvenir. Partis de leur propre expérience de marcheurs des $\mathrm{XX}^{\mathrm{e}}$ et $\mathrm{XXI}^{\mathrm{e}}$ siècles, des chercheurs fondent cette interrogation sur le postulat selon lequel la route, chemin matériel, est le lien évident qui relie l'hôpital et le pèlerin.

Un examen attentif des archives hospitalières de Lille oblige pourtant à remettre en cause cette certitude et à s'interroger sur le bien-fondé de cette question de l'antériorité de la route sur l'hôpital. La manipulation de ce matériau nécessite quelques précautions. Tous les hôpitaux n'accueillent pas les pèlerins, malgré les préceptes divins exposés dans l'Évangile de Matthieu'. À l'instar d'autres villes, Lille connaît au XIII ${ }^{\mathrm{e}}$ siècle une véritable «floraison hospitalière » selon l'expression de Michel Mollat ${ }^{6}$, conséquence de la «révolution de la charité » décrite par André Vauchez ${ }^{7}$. Les prédicateurs incitent riches et puissants à fonder des hôpitaux pour y accomplir une partie des œuvres de miséricorde. La comtesse de Flandre, Jeanne de Constantinople,

Lande: histoire naturelle et géographie historique. Actes du Colloque de Sabres (27-29 novembre 1981), éd. CNRS et Parc Naturel Régional des Landes de Gascogne, 1985, p. 177-196.

5 «Quand le Fils de l'homme viendra dans sa gloire, escorté de tous les anges, alors il prendra place sur son trône de gloire. Devant lui seront rassemblées toutes les nations, et il séparera les gens les uns des autres, tout comme le berger sépare les brebis des boucs. Il placera les brebis à sa droite et les boucs à sa gauche. Alors le Roi dira à ceux de droite: "Venez les bénis de mon Père, recevez en héritage le Royaume qui vous a été préparé depuis la fondation du monde. Car j'ai eu faim et vous m'avez donné à manger, j'ai eu soif et vous m'avez donné à boire, j'étais un étranger et vous m'avez accueilli, nu et vous m'avez vêtu, malade et vous m'avez visité, prisonnier et vous êtes venus me voir". Alors les justes lui répondront: "Seigneur, quand nous est-il arrivé de te voir affamé et de te nourrir, assoiffé et de te désaltérer, étranger et de t'accueillir, nu et de te vêtir, malade ou prisonnier et de venir te voir ?" Et le Roi leur fera cette réponse : "En vérité je vous le dis, dans la mesure où vous l'avez fait à l'un de ces plus petits de mes frères, c'est à moi que vous l'avez fait" " (Matthieu, XXV, 31-40, dans la Bible de Jérusalem).

${ }^{6}$ Il s'agit du titre du deuxième chapitre rédigé par Michel Mollat dans la synthèse établie sous la direction de Jean IMBERT, $L$ 'Histoire des hôpitaux en France, Toulouse, Privat, 1982.

7 André VAucheZ, "Assistance et charité en Occident, XIII ${ }^{\mathrm{e}}-\mathrm{XV}^{\mathrm{e}}$ siècles », in Vera BARBAGLi BAGNOLI (dir.), Domanda e consumi. Livelli e strutture (nei secoli XIII-XVIII). Atti della sesta settimana di studio (27 aprile-3 maggio 1974) dell' Istituto internazionale di storia economica F. Datini, Prato, Florence, L.S. Olschki, «Atti delle settimane di studio e altri convegni », 6, 1978, p. 151-162 (cit. p. 152). 
entend alors l'ensemble des recommandations du Christ et fonde en 1233 l'Hôpital Saint-Sauveur et en 1237 l'Hôpital Notre-Dame, surnommé par la suite Comtesse par les Lillois. Deux siècles plus tard, à la demande de son épouse Isabelle de Portugal, le duc de Bourgogne Philippe le Bon fonde l'Hôpital Saint-Jacques. Seules ces trois maisons répondent exactement aux préceptes divins. Les onze autres hôpitaux de la ville, fondés entre les XIII ${ }^{\mathrm{e}}$ et $\mathrm{XV}^{\mathrm{e}}$ siècles, n'accueillent pas les pèlerins, perçus avant tout comme des étrangers, mais tout au plus les pauvres passants connus par la population qui vit à l'intérieur des remparts. D'emblée, la recherche doit se concentrer sur les trois hôpitaux comtaux. Il faut par ailleurs conserver à l'esprit qu'un hôpital médiéval n'est pas seulement un lieu d'accueil et de soins des pauvres, des malades et des pèlerins, mais avant tout une communauté d'hommes et de femmes en marche vers le Salut, selon la volonté des fondateurs. Les accueillis doivent se soumettre à des rites d'entrée - non dénués d'aspects pratiques - comme le mandé ou lavement des pieds et la confession. Tout le temps que dure leur séjour, ils font partie de la communauté religieuse. Celle-ci est donc constituée de membres temporaires et d'autres permanents. Ces derniers ont souvent été oubliés par les historiens qui se sont avant tout intéressés aux accueillis. Les archives des hôpitaux comtaux, et surtout celles de l'Hôpital Comtesse, permettent de rétablir cet équilibre.

Il devient alors possible d'appréhender la diversité des pèlerins. Sont-ils tous des «marcheurs de Dieu ${ }^{8}$ accomplissant leur périple religieux dans la souffrance du corps et le dénuement le plus complet ? L'épreuve physique et son support - la route - sont-ils au Moyen Âge l'aspect le plus marquant du voyage ? Peut-on penser que la route et son tracé sont ce qui relie de manière évidente le pèlerin et l'hôpital ?

\footnotetext{
${ }^{8}$ Nous reprenons l'expression de Pierre-André SigAL, Les Marcheurs de Dieu : pèlerinages et pèlerins au Moyen Âge, Paris, A. Colin, «U prisme », 1974.
} 


\section{Une minorité de pèlerins aux portes des hôpitaux lillois}

Les hôpitaux comtaux de Lille reçoivent les pèlerins qui se présentent à leur porte, ce qui n'est pas le cas de tous. On peut même raisonnablement penser que ces maisons n'accueillent qu'une minorité d'entre eux. Avant d'esquisser leurs portraits, il convient de revenir sur l'indistinction entre pèlerin et malade, effective dans certains hôpitaux médiévaux. Un certain nombre d'études d'histoire hospitalière s'attachent à distinguer les différents groupes de personnes qui y sont accueillis ${ }^{9}$. Cette préoccupation est celle de 1'historien qui tente de rendre compréhensible à son contemporain le fonctionnement d'un établissement régi par un système de pensée différent du sien et pour qui un hôpital doit regrouper en un même service les patients atteints de pathologies proches pour des raisons pratiques et sanitaires. Il n'y a pas lieu pour les comtes de Flandre puis les ducs de Bourgogne de séparer alités et marcheurs, et ce pour plusieurs raisons. D'une part, les fondateurs sont mus par des motifs religieux : l'Évangile de Matthieu rapporte que le Christ fait des malades et des voyageurs ses frères et sœurs sans les différencier. Ils suivent à la lettre l'enseignement du Christ à des fins sotériologiques. D'autre part, et cette deuxième raison ne saurait être dissociée de la première, les comtes s'affirment ainsi en protecteurs de l'ensemble des habitants et des voyageurs en Flandre. Les bourgeois et les

\footnotetext{
9 Voir par exemple Jacqueline CAILlE, Hôpitaux et charité publique à Narbonne au Moyen Âge: de la fin du $\mathrm{XI}^{\mathrm{e}}$ à la fin du $\mathrm{XV}^{\mathrm{e}}$ siècle, Toulouse, Privat/Société Française d'Histoire des Hôpitaux, 1978, dont la partie intitulée «Typologie des assistés » (p. 100-104) s'ouvre sur ces mots : «nous distinguerons trois catégories : les malades, les pauvres, les pèlerins » (p. 100) et Annie SAUNIER, «Le pauvre malade» dans le cadre hospitalier médiéval : France du Nord, vers 1300-1500, Paris, Arguments, 1993. Annie Saunier établit ainsi une typologie qui distingue «admissions à caractères sociaux » et «admissions à caractère médical ». Nous renvoyons enfin aux préconisations de François-Olivier TouATI, «Un dossier à rouvrir : l'assistance au Moyen Âge », in Henri Platelle et Jean Dufour (dir.), Fondations et ouvres charitables au Moyen Âge. $121^{\mathrm{e}}$ Congrès national des sociétés historiques et scientifiques, Section d'histoire médiévale et philologie, Nice, 26-31 octobre 1996, Paris, Comité des travaux historiques et scientifiques, 1999, p. 23-38 (p. 33).
} 
chanoines de Lille font un choix différent : leurs «prochains » sont avant tout leurs « pairs $»^{10}$. Enfin, les actes médicaux accomplis par des professionnels - médecins, barbiers, chirurgiens, apothicaires - sont encore rares. Les besoins des malades se rapprochent alors beaucoup de ceux des pèlerins. Le pieux voyageur et le malade sont deux figures différentes du Seigneur qui fait d'eux ses préférés, ceux qui lui ressemblent le plus. Tous deux quittent leur foyer et cheminent dans la douleur du corps vers le Christ. Le pèlerin prend la route en direction d'un sanctuaire. Le malade amené à l'hôpital est souvent dans un état critique et peut se sentir rappelé à Dieu. Il n'est pas rare que le pèlerin, lorsqu'il part au loin et doute de son retour, établisse un testament avant son départ. Le malade peut faire de même.

Tous deux arrivent donc à l'hôpital avec les mêmes besoins : repos, chaleur en hiver, nourriture reconstituante tant pour le corps que pour l'âme, afin de pouvoir poursuivre d'une manière ou d'une autre leur propre chemin. La sensation d'isolement est parfois grande. Loin de chez lui, le marcheur de Dieu peut se sentir déraciné. Quant au malade gravement atteint, son calvaire personnel peut lui donner un fort sentiment de solitude. L'encadrement spirituel est donc primordial afin de rassurer ces deux voyageurs. Les hôpitaux comtaux disposent de leur propre chapelle avec un desservant pour dire les messes. Un prêtre est présent pour recueillir les confessions et soulager les âmes de leurs péchés. Les corps ne sont cependant pas négligés. Les comptes font apparaître

\footnotetext{
${ }^{10}$ C'est principalement le cas des hôpitaux fondés avant le XIV ${ }^{\mathrm{e}}$ siècle. L'Hôpital des Clercs, tenu par le Chapitre Saint-Pierre, n'accueille que les chanoines et leurs serviteurs, tandis que les hôpitaux municipaux Saint-Nicolas, Saint-Nicaise et de la Trinité sont réservés aux bourgeois. Saint-Nicolas reçoit cependant ceux qui, faute de moyens financiers, ont été déchus de leurs privilèges. En 1321, l'hôpital Saint-Julien est fondé par la veuve d'un bourgeois et administré par les échevins afin de recevoir des pauvres pour la nuit. Cependant, en 1468, au moment où s'ouvre cette étude, Saint-Julien permet avant tout aux échevins et au duc de distribuer des prébendes (cf. Archives Municipales de Lille, AA 197, pièce 3961). Les hôpitaux des Grimaretz et Saint-Jean-Baptiste, fondés au XV siècle, accueillent les pauvres sans distinction de statut et n'ont pas pour mission de recevoir les pèlerins de passage. L'hôpital des Marthes accueille huit pauvres femmes âgées de plus de cinquante ans. La Maison des Bons Enfants est un orphelinat.
} 
une nourriture abondante et sans aucun doute plus diversifiée que dans l'ordinaire du pauvre. La viande, bovine et porcine, les volailles, les produits sucrés, les fruits constituent les principaux reconstituants offerts aux hôtes. En elle-même, cette alimentation exceptionnelle est un remède contre les maladies qui touchent les corps affaiblis par les travaux harassants et la sous-nutrition ou la malnutrition. Il ne s'agit pas pour l'hôpital d'offrir au malade ou au pèlerin une occasion de faire bombance, mais seulement de lui donner la force de poursuivre sa route. Les hôpitaux constituent des étapes sur la route qui mène au Seigneur.

Les pèlerins sont accueillis quelles que soient leur provenance et leur destination. Il importe de préciser que le pèlerinage lointain n'est pas la coutume. Seule la duchesse de Bourgogne qui dédie sa fondation au saint galicien précise que celle-ci doit accueillir «pelerins passans venans de mondit seigneur saint Jacques de Galice, y alans aussi et d'aultres pelerinages quelconques ${ }^{11}$. Monique Sommé a fait l'hypothèse de la volonté de la duchesse, d'origine ibérique, de développer ce pèlerinage ${ }^{12}$. Dans le Nord, il n'est pas nécessaire de se rendre à Compostelle pour honorer les reliques de l'apôtre: d'autres sanctuaires, plus proches, comme la collégiale Saint-Pierre d'Aire-sur-la-Lys ou l'abbaye Saint-Vaast d'Arras organisent un culte autour des deux parties du chef de saint Jacques qu'elles se sont âprement disputées entre les $\mathrm{XI}^{\mathrm{e}}$ et $\mathrm{XIII}^{\mathrm{e}}$ siècles ${ }^{13}$. La piété mariale est également très forte. Depuis 1270, la ville de Lille est le siège d'une importante procession à Notre-Dame de la Treille : il n'est pas rare que des habitants des villes environnantes se joignent aux Lillois pour célébrer la Vierge en cette occasion. En plus de ces sanctuaires,

\footnotetext{
${ }^{11}$ Lille, Archives Départementales du Nord (ADN), AH10/A1, pièce n ${ }^{\circ}$.

${ }^{12}$ Monique Somme, Isabelle de Portugal, duchesse de Bourgogne. Une femme au pouvoir au $X V^{e}$ siècle, Lille, Presses universitaires du Septentrion, "Histoire et civilisations », 1998, p. 463.

${ }^{13}$ Cf. Denise PERICARD-MEA, «Le culte de saint Jacques dans les Flandres », in Georges JEHEL (dir.), Orient et Occident du IX ${ }^{\mathrm{e}}$ au XV siècle. Actes du colloque d'Amiens des 8, 9 et 10 octobre 1998, Paris, Éditions du Temps, «Questions d'histoire », 2000, p. 71-80.
} 
les chapelles des établissements comtaux disposent de reliques, parfois importantes comme celles de saint Augustin $^{14}$, et leurs dirigeants obtiennent des indulgences pour ceux qui viendraient se rendre en pèlerinage dans ces chapelles ${ }^{15}$.

Les pèlerins qui se présentent aux portes de l'hôpital sont généralement pauvres, à moins qu'ils ne souhaitent, dans un esprit exceptionnel de dévotion, voyager dans les conditions des plus démunis. Le pèlerinage ne contraint pas forcément le marcheur à l'ascèse. Le séjour dans les hôtels ne lui est pas interdit et Lille n'en est pas dépourvue. Les archives hospitalières évoquent au moins un hôtel, celui de 1'Angele, place Saint-Martin ${ }^{16}$. Entre février 1488 et mars 1489, Jean de Tournai, un riche marchand de Valenciennes, se rend en pèlerinage à Rome, Jérusalem et Compostelle - entre autres. Dès qu'il le peut, il fait étape dans les meilleures auberges, comme celle du Lion Blanc à Venise. À Jérusalem, il ne loge pas à l'hospice des pèlerins ${ }^{17}$. Ce type de pieux voyageur ne s'arrête probablement à l'Hôpital Comtesse ou à Saint-Jacques que de mauvaise grâce. De plus, aucun don ou legs opéré par des voyageurs de Dieu ne semble avoir laissé de traces écrites dans les archives pourtant bien fournies de ces maisons. On peut donc en déduire que ceux qui s'arrêtent dans ces maisons charitables sont réellement pauvres. S'ils font un don, celui-ci est trop faible pour valoir la peine d'être consigné dans les layettes hospitalières.

Les marcheurs se déplacent seuls ou en tout petits groupes. $\mathrm{Au} \mathrm{XV}$ siècle, l'Hôpital Comtesse dispose d'une petite trentaine de lits au maximum ${ }^{18}$. L'Hôpital Saint-Jacques ne rémunère que deux servantes au service des

\footnotetext{
${ }^{14}$ Lille, ADN, AH1/557.

${ }^{15}$ Lille, ADN, AH1/673.

${ }^{16}$ Lille, ADN, AH1/4427, 71v.

${ }^{17}$ Cf. Lucie PolaK, «Un récit de pèlerinage de 1488-1489 », Le Moyen Âge, 87 (1981), p. 71-88 (p. 82).

${ }^{18}$ Cf. Laurent SEvin, L'Hôpital Notre-Dame de Lille des origines à 1317, Mémoire de maîtrise, Lille, Université Lille III, 1997, p. 154-155.
} 
parturientes et des pèlerins ${ }^{19}$. Aucun des deux n'est donc en mesure de recevoir un grand nombre de pèlerins à la fois. C'est alors aux échevins de la ville de Lille que revient la tâche de les accueillir. En 1457, ils ouvrent la halle échevinale à un groupe de deux-cents soixante pèlerins allemands en route vers le Mont-Saint-Michel ${ }^{20}$. Ils leur fournissent de la paille, du bois et quelques subsides. Il s'agit probablement de gérer là un événement qui pourrait se muer rapidement en trouble à l'ordre public - le souvenir du passage des flagellants au $\mathrm{XIV}^{\mathrm{e}}$ siècle est encore vif ${ }^{21}$.

Les hommes n'ont pas le monopole du pèlerinage. Un acte de 1444 rappelle en effet que la duchesse de Bourgogne a fondé cet hôpital pour «l'usage des pelerins et pelerines allant et revenant du voyage de SaintJacques $»^{22}$. Les femmes en pèlerinage font bien sûr l'objet de railleries : Chaucer ou Rutebeuf ont dénoncé ces femmes qui s'amusent beaucoup plus qu'elles ne prient en chemin ${ }^{23}$. Ces critiques relèvent du topos misogyne de la femme sensuelle et perverse en toute occasion; toutefois, Isabelle de Portugal cherche sans doute à offrir aux femmes un hébergement où leur vertu ne serait pas en danger. Sa fondation leur est particulièrement destinée : l'Hôpital SaintJacques reçoit également les parturientes. L'établissement mène de front ces deux missions et il ne nous semble pas exact de penser, comme Denis Clauzel $^{24}$,

${ }^{19}$ Cf. Joseph WARICHEZ, «État bénéficial de la Flandre et du Tournaisis », Analectes pour servir à l'histoire ecclésiastique de la Belgique, 35 (1909), p. 254-255, cité dans Monique Somme, Isabelle de Portugal, duchesse de Bourgogne..., op. cit., p. 462.

${ }^{20}$ Cf. Archives historiques et littéraires du Nord de la France, $3^{\mathrm{e}}$ série, t. IV, p. 308, cité dans Histoire de Lille, Louis TRENARD (dir.), Lille/Toulouse, R. Giard/Privat, 1970, p. 408.

${ }^{21}$ La peste qui sévit à partir de 1348 est rapidement perçue comme une punition divine. Afin d'apaiser la colère de Dieu, des groupes de pèlerins se forment pour expier leurs fautes dans l'imitation du Christ. Les flagellants parcourent ainsi les routes pendant trente-trois jours et demi (l'âge du Christ) tout en appliquant à leur corps de violentes mortifications. Peu à peu, des idées anticléricales apparaissent au sein de ces groupes. Le mouvement a pris une grande ampleur dans les villes du Nord.

${ }^{22}$ Lille, ADN, AH10/A1, $2^{\text {e }}$ pièce, vidimus de 1514 d'un acte du 28 juillet 1444.

${ }^{23}$ Cf. Denis Clauzel, «Les faux pèlerins à l'automne du Moyen Âge », in Voyageurs et pèlerins de l'Antiquité à nos jours, Mélanges de science religieuse, 66, n⿳3 3 (2009), p. 69-88 (p. 74).

${ }^{24}$ Ibid., p. 83. 
que la charge obstétrique ait été imposée par les échevins pour amoindrir les possibilités d'accueil des pèlerins. En tous les cas en 1455, d'après l'état bénéficial réalisé sur l'ordre de l'évêque de Tournai, et malgré les difficultés financières de la maison charitable, celle-ci s'adonne toujours aux deux tâches qui lui ont été confiées par la duchesse ${ }^{25}$. Femme d'action, régulièrement en déplacement, Isabelle de Portugal agit peut-être par solidarité envers celles de son sexe qu'elle sait malmenées de bien des façons.

Souvent pauvres, hommes, plus rarement femmes, voyageant seuls ou en petits groupes, les pèlerins accueillis dans l'un des hôpitaux comtaux lillois méritent sans doute leur surnom de «marcheurs de Dieu ». Mais ils ne sont pas représentatifs de l'ensemble des pèlerins. Les archives de l'Hôpital Comtesse nous laissent entrevoir l'existence d'autres pieux voyageurs, moins démunis mais aussi moins anonymes et qui ne se déplacent pas uniquement à pied : les religieux et religieuses de la communauté hospitalière de l'Hôpital Comtesse.

\section{Des pèlerins particuliers : les frères et sœurs de l'Hôpital Comtesse}

L'établissement fondé par Jeanne de Constantinople en 1237 n'est pas seulement une étape au cours d'un pèlerinage ou une destination pour celui qui souhaite recevoir les indulgences prévues lors de la visite de l'une des chapelles de la charitable maison. L'Hôpital Comtesse est aussi le point central à partir duquel rayonnent les chemins de pèlerinage d'une communauté religieuse impliquée dans toutes les affaires du siècle, qu'elles soient politiques, économiques ou sociales. On peut s'étonner devant le nombre de pèlerinages accomplis par les membres soignants de la communauté hospitalière. Tous les jours, ils accomplissent une grande part des œuvres de miséricorde nécessaires au Salut. Le pèlerinage n'est pas pour les chrétiens une obligation, comme c'est

${ }^{25}$ Cf. Monique Somme, Isabelle de Portugal, duchesse de Bourgogne..., op. cit., p. 462. 
le cas pour les musulmans. Pourtant, sur la période étudiée - de $1467^{26}$ à $1520^{27}$ - près de la moitié des comptes attestent l'accomplissement d'un voyage de dévotion ${ }^{28}$. Certaines années, trois mentions de voyages à destination de sanctuaires peuvent être relevées, comme en $1468-1469^{29}$ et en $1498-1499^{30}$. Alors qu'ils sont au plus près des préférés du Christ et s'en font les humbles serviteurs, et tandis qu'ils ont en leurs murs des chapelles attirant les fidèles en quête d'indulgences, les religieux de l'Hôpital prennent la route pour se rapprocher de Dieu : on ne peut que s'interroger sur les motivations de ces pèlerins particuliers. Répondre à cette interrogation requiert de dresser la carte de leurs lieux de pèlerinages ${ }^{31}$.

L'abbaye bénédictine de Notre-Dame de Messines, au nord-ouest de Lille, apparaît seize fois ${ }^{32}$ comme lieu de pèlerinage. Ces mentions sont régulièrement réparties au cours de la période observée. Viennent ensuite Saint-Adrien de Grammont (huit fois) ${ }^{33}$, Saint-Druon de Cambrai (cinq fois) ${ }^{34}$, Saint-Piat de Seclin (quatre fois) ${ }^{35}$, Saint-Maurand de Douai (quatre fois) ${ }^{36}$, Sainte-Aragone

\footnotetext{
${ }^{26}$ Lille, ADN, AH1/4397. Les comptes antérieurs ont été détruits dans l'incendie de 1467 qui, en une nuit, a ravagé l'établissement.

${ }^{27}$ Lille, ADN, AH1/4482. En 1519-1520 meurt Nicole De Le Carnoye, maître charismatique de l'Hôpital Comtesse depuis 1496.

${ }^{28}$ Les comptes signés par les visiteurs (ou contrôleurs) de la maison et dans les livresjournaux consignent les frais de déplacement des religieux. La destination assortie du mot « pèlerinage » ou la mention d'une offrande ont retenu notre attention.

${ }^{29}$ Lille, ADN, AH1/4399, fol. $37 v^{\circ}$.

${ }^{30}$ Lille, ADN, AH1/4441, fol. $42 \mathrm{v}^{\circ}$ et $43 \mathrm{v}^{\circ}$.

${ }^{31}$ Voir carte en annexe, p. 64. Les sanctuaires de Saint-Quentin, Sainte-Radegonde et SaintMartin près de Péronne, bien plus au sud de l'espace cartographié, n'ont pas été représentés car ils ne sont pas situés dans l'aire d'action de l'Hôpital Comtesse.

32 Lille, ADN, AH1/4408, fol. 53v ; AH1/4411, fol. $62 \mathrm{v}^{\circ}$; AH1/4427, fol. 40v $\mathrm{v}^{\circ}$ (deux

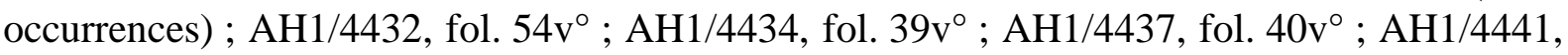
fol. $42 \mathrm{v}^{\circ}$ (deux occurrences), fol. $43 \mathrm{r}^{\circ} ; \mathrm{AH} 1 / 4444$, fol. $63 \mathrm{v}^{\circ} ; \mathrm{AH} 1 / 4447$, fol. $39 \mathrm{v}^{\circ}$;

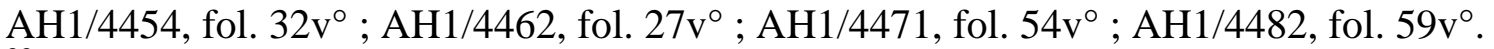

${ }^{33}$ Lille, ADN, AH1/4399, fol. 34v $\mathrm{v}^{\circ}$; AH1/4403, fol. 41r ${ }^{\circ}$; AH1/4408, fol. 53v $\mathrm{v}^{\circ}$; AH1/4411, fol. $62 \mathrm{v}^{\circ}$; $\mathrm{AH} 1 / 4416$, fol. $44 \mathrm{v}^{\circ}$; $\mathrm{AH} 1 / 4427$, fol. $71 \mathrm{r}^{\circ}$; $\mathrm{AH} 1 / 4436$, fol. 53r $\mathrm{r}^{\circ}$, AH1/4437, fol. $40 \mathrm{v}^{\circ}$.

${ }^{34}$ Lille, ADN, AH1/4427, fol. 71r ${ }^{\circ}$; AH1/4433, fol. 57r ${ }^{\circ}$; AH1/4436, fol. 53r ${ }^{\circ}$; AH1/4449, fol. 40r ; AH1/4482, fol. $59 \mathrm{v}^{\circ}$.

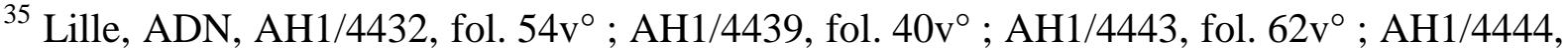
fol. $63 v^{\circ}$.
} 
(ou Radegonde) et Saint-Martin près de Péronne (trois fois) ${ }^{37}$. Notre-Dame de $\mathrm{Hal}^{38}$, Saint-Nazaire à Ablain ${ }^{39}$ et Saint-Quentin ${ }^{40}$ près de Péronne reviennent deux fois, tandis que Saint-Antoine de Bailleul ${ }^{41}$ et Saint-Vincent de Soignies ${ }^{42}$ ne font l'objet que d'une mention unique. Cette carte appelle plusieurs remarques. D'une part, les pieux déplacements des frères et sœurs se font dans un rayon de quatre-vingt-dix kilomètres. Le pèlerinage lointain n'est pas de mise : Rome n'est qu'une destination «pour les besongnes et affaires dudit hospital $»^{43}$. D'autre part, le temps passé loin de l'Hôpital n'excède jamais huit jours. La préférence des religieux va à des voyages de piété courts mais régulièrement renouvelés. Par ailleurs, la carte des lieux de pèlerinage de la communauté de l'hôpital ne coïncide pas avec celle de ses possessions foncières ou encore de son influence politique et sociale par le biais de la possession du droit de vent ${ }^{44}$. Il n'est pas non plus question de complaire aux ducs de Bourgogne, successeurs des comtes de Flandre et autorité suprême de la maison charitable. En effet, seuls Saint-Adrien de Grammont et Notre-Dame de Hal correspondent à des sanctuaires qui ont été régulièrement fréquentés par les ducs de Bourgogne ${ }^{45}$, mais à une période un peu antérieure à celle étudiée grâce aux

36 Lille, ADN, AH1/4436, fol. 53v ; AH1/4445, fol. $61 \mathrm{v}^{\circ}$; AH1/4451, fol. $39 \mathrm{v}^{\circ}$ (deux occurrences).

${ }^{37}$ Lille, ADN, AH1/ 4449, fol. 39v $\mathrm{v}^{\circ}$; AH1/4451, fol. 39v (deux occurrences).

${ }^{38}$ Lille, ADN, AH1/4399, fol. 37v $\mathrm{v}^{\circ}$; AH1/4408, fol. 53v ${ }^{\circ}$.

${ }^{39}$ Lille, ADN, AH1/4411, fol. $53 \mathrm{v}^{\circ}$; AH1/4421, fol. $49 \mathrm{r}^{\circ}$.

${ }^{40}$ Lille, ADN, AH1/4451, fol. $39 v^{\circ}$; AH1/4457, fol. $28 v^{\circ}$.

${ }^{41}$ Lille, ADN, AH1/4399, fol. $37 \mathrm{v}^{\circ}$.

${ }^{42}$ Lille, ADN, AH1/4437, fol. 40v ${ }^{\circ}$.

${ }^{43}$ Lille, ADN, AH1/4439, fol. $41 \mathrm{r}^{\circ}$.

${ }^{44}$ En 1243, Jeanne de Constantinople fait don à l'Hôpital Notre-Dame de Lille de tous ses moulins et du droit de vent (Lille, ADN, AH1/27). Dans les limites mal définies d'un espace appelé « mannée de Lille », aucun moulin ne peut donc être érigé sans l'accord de l'Hôpital Comtesse. Ce privilège extrêmement important contraint tous les détenteurs de moulins, quelle que soit leur puissance économique et politique, à négocier avec la maison charitable pour obtenir le droit d'utiliser les précieuses machines.

${ }^{45}$ Cf. Bertrand SCHNERB, «La piété et les dévotions de Philippe le Bon, duc de Bourgogne (1419-1467) », Comptes-rendus des séances de l'année, Académie des Inscriptions et BellesLettres, 149, $\mathrm{n}^{\circ} 4$ (2005), p. 1319-1344 (p. 1336), et Monique SoMME, Isabelle de Portugal, duchesse de Bourgogne..., op. cit., p. 454. 
comptes. Si l'objectif de ces dévotions avait été politique, l'année où Nicole De Le Carnoye devient maître de l'hôpital ${ }^{46}$ n'aurait pas marqué la fin de ces pratiques, ce qui est pourtant le cas. Ces pèlerinages ne font donc que répondre à un besoin spirituel et religieux émanant des soignants, et qu'il convient de préciser.

La mission quotidienne des religieux et religieuses de l'Hôpital Comtesse en fait les serviteurs des préférés du Christ. Cela n'efface cependant pas les inquiétudes sotériologiques des frères et sœurs et de tous ceux qui travaillent à leurs côtés. On peut en réalité distinguer deux types de pèlerinage, individuel et collectif. En effet, l'appartenance à la communauté hospitalière n'empêche pas les religieux de conserver une certaine autonomie cultuelle, surtout lorsque leur santé est menacée. Les pèlerinages d'un religieux nommé Jehan Toelins occupent ainsi une place particulière dans cette étude. Au cours des années 1504,1505 et 1506, le frère accomplit trois déplacements à Sainte-Aragone et Saint-Martin, près de Péronne. Il s'y rend en charrette, accompagné par un serviteur. Il s'agit d'un des périples les plus lointains. Ce sont les seules mentions de ces deux sanctuaires au cours des cinquante-trois années étudiées. Jehan Toelins est l'héritier présomptif du maître Nicole De Le Carnoye. Le prédécesseur de ce dernier, Eloi de Fâches, avait déjà pris soin de financer les études du jeune homme. En 1504, l'Hôpital Comtesse fait appel au savoir-faire médical des sœurs de Saint-Sauveur afin de concevoir des onguents pour le frère souffrant et incapable de se mouvoir. En 1506, ce dernier accomplit un dernier voyage à Sainte-Radegonde et Saint-Martin. Il reprend ensuite ses déplacements d'affaires avec le maître. Il y a donc toutes les raisons de penser qu'il s'agit là de pèlerinages votifs. Le malade est allé toucher la pierre de sainte Radegonde et le grès de saint Martin, réputés pour leurs qualités thaumaturgiques ${ }^{47}$. Sa quête est celle de la santé individuelle et non celle du Salut collectif.

\footnotetext{
${ }^{46}$ C'est-à-dire l'année 1496-1497.

${ }^{47}$ Cf. G. Boulanger, « La Pierre de Sainte-Radegonde et le Grès de Saint-Martin », Bulletin
} 
Les pèlerinages à Saint-Adrien, à Notre-Dame de Messines ou à Saint-Quentin relèvent de cette dernière logique. Le groupe de pieux voyageurs représente la communauté hospitalière dans son entier et dans toute sa diversité. Il est donc guidé par l'un de ses membres éminents, le maître, la prieuse, ou un frère régulièrement chargé des affaires importantes de l'Hôpital. Les sœurs, souvent au nombre de trois, prennent la route comme les frères. Le nombre de religieuses ayant reçu des soins dans l'année ne correspond pas systématiquement à celui des pèlerines. Il arrive aussi que les religieux emmènent en tant que pèlerins - et non en tant que simples serviteurs - des familiers de la maison ${ }^{48}$. Le choix répété de donner en offrande à Saint-Quentin le contrepoids en cire de la prieuse ${ }^{49}$ ne fait que confirmer la volonté des frères et sœurs en déplacement de placer sous la protection du saint l'ensemble de la communauté hospitalière. Les soignants restés auprès des malades bénéficient ainsi du voyage de piété sans avoir pris la route. Le pèlerinage par procuration n'est d'ailleurs pas une pratique isolée chez les gens aisés et l'Hôpital Comtesse y recourt également. En 1504-1505, une «povre dame pieuse » est envoyée à Saint-Druon. Les frais de son pèlerinage sont pris en charge par la maison charitable $^{50}$. Ce n'est en rien une aumône, comme celle que les échevins de la ville de Lille accordent parfois à un pauvre pèlerin incapable de payer avec ses propres deniers son contrepoids en cire ${ }^{51}$, mais une manière d'assurer le Salut de la communauté hospitalière.

La responsabilité des âmes qui vivent dans les murs de l'Hôpital incombe au maître. Il n'est donc pas surprenant que l'itinéraire spirituel de l'établissement reflète celui de son directeur. Les changements de lieu de culte sont d'ailleurs sensibles et imputables directement aux renouvellements des

de la Société préhistorique française, 15, n5 (1918), p. 279-283.

${ }^{48}$ Lille, ADN, AH1/4408, fol. 53v .

${ }^{49}$ Lille, ADN, AH1/4451, fol. $39 v^{\circ}$; AH1/4458, fol. $28 v^{\circ}$.

${ }^{50}$ Lille, ADN, AH1/4449, fol. 40r ${ }^{\circ}$.

${ }^{51}$ Cf. Louis Trenard, Histoire de Lille ..., op. cit., p. 409. 
dirigeants. Saint-Adrien de Grammont est le sanctuaire de prédilection d'Eloi de Fâches. Son successeur, Nicole De Le Carnoye ne poursuit pas cette tradition mais reprend et accentue volontiers celle de se rendre à Messines. Il ne s'agit pas seulement de se rendre à la procession annuelle qui est faite autour des reliques de la sainte Croix ${ }^{52}$, puisqu'en 1498-1499 différents membres de la maison charitable s'y rendent en pèlerinage en trois groupes à des moments différents $^{53}$. Nicole De Le Carnoye fait le choix de placer la communauté qui lui a été confiée sous la protection des plus hauts intercesseurs, la Vierge et le Christ. Les pèlerinages accomplis par les frères et sœurs sont en réalité le prolongement logique des tâches qu'ils accomplissent tous les jours auprès des pauvres du Seigneur. Ces pieux voyages sont nécessaires à la guérison des âmes et des corps et constituent des remèdes au même titre que les onguents commandés aux apothicaires et les messes dites dans la chapelle de la maison.

Toutefois les conditions pénibles dans lesquelles cheminent les «marcheurs de Dieu » accueillis à l'Hôpital Comtesse ne semblent pas être nécessaires au bon déroulement d'un pèlerinage. En effet, les frères et sœurs de l'Hôpital Comtesse accomplissent leurs dévotions sans grande souffrance. Ils sont souvent en chariot $^{54}$, accompagnés d'un ou de plusieurs serviteurs. L'épreuve physique est réduite à sa plus simple expression - les cahots du transport - et le risque de faire de mauvaises rencontres relativement amoindri. Parvenus au terme de leur périple, les religieux ont les moyens financiers de rendre au saint le culte qui lui est dû.

\footnotetext{
${ }^{52}$ En 1467 et 1469, l'abbaye commande trois reliquaires en argent, dont un ayant la forme des tours de l'église de Messines. Les religieuses souhaitent probablement raviver le culte autour de ces reliques. Cf. Monasticon Belge, III, Province de Flandre occidentale, Denée/Liège, Abbaye de Maredsous/Centre national de recherches d'histoire religieuse, 1960-1978, vol. 1, p. 218.

${ }^{53}$ Lille, ADN, AH1/4441, fol. $42 \mathrm{v}^{\circ}$ (deux occurrences) et fol. $43 \mathrm{r}^{\circ}$.

${ }^{54}$ Lille, ADN, AH1/ 4408, fol. 53v $\mathrm{v}^{\circ}$; AH1/4411, fol. $62 \mathrm{v}^{\circ}$; AH1/4434 fol. 39v $\mathrm{v}^{\circ}$; AH1/4445, fol. $61 v^{\circ}$; AH1/4449 fol. 39v $\mathrm{v}^{\circ}$; AH1/4451, fol. 39v ${ }^{\circ}$; AH1/4454, fol. 32r ${ }^{\circ}$; AH1/4457, fol. $28 \mathrm{v}^{\circ}$; AH1/4462, fol. 27v $\mathrm{v}^{\circ}$ AH1/4482, fol. $59 \mathrm{v}^{\circ}$.
} 
À mi-chemin entre un Jean de Tournai et un pauvre hère en quête de Dieu, le religieux de l'Hôpital Comtesse apparaît comme un autre type de pieux voyageur, qui ne saurait être qualifié de marcheur. Pourtant, tous trois sont des pèlerins et parcourent des routes pour atteindre un sanctuaire. Les pieds endoloris ne sont pas une condition nécessaire à l'accomplissement de cette pratique pieuse. À l'issue de cette étude, on ne peut pas nier que la route est le lien fondamental et évident entre le pèlerin et l'hôpital, à condition de préciser que cette route n'est en rien matérielle, mais bien spirituelle : il s'agit de celle qui mène le pèlerin à son Salut et dont il serait bien difficile d'établir le tracé sur le papier.

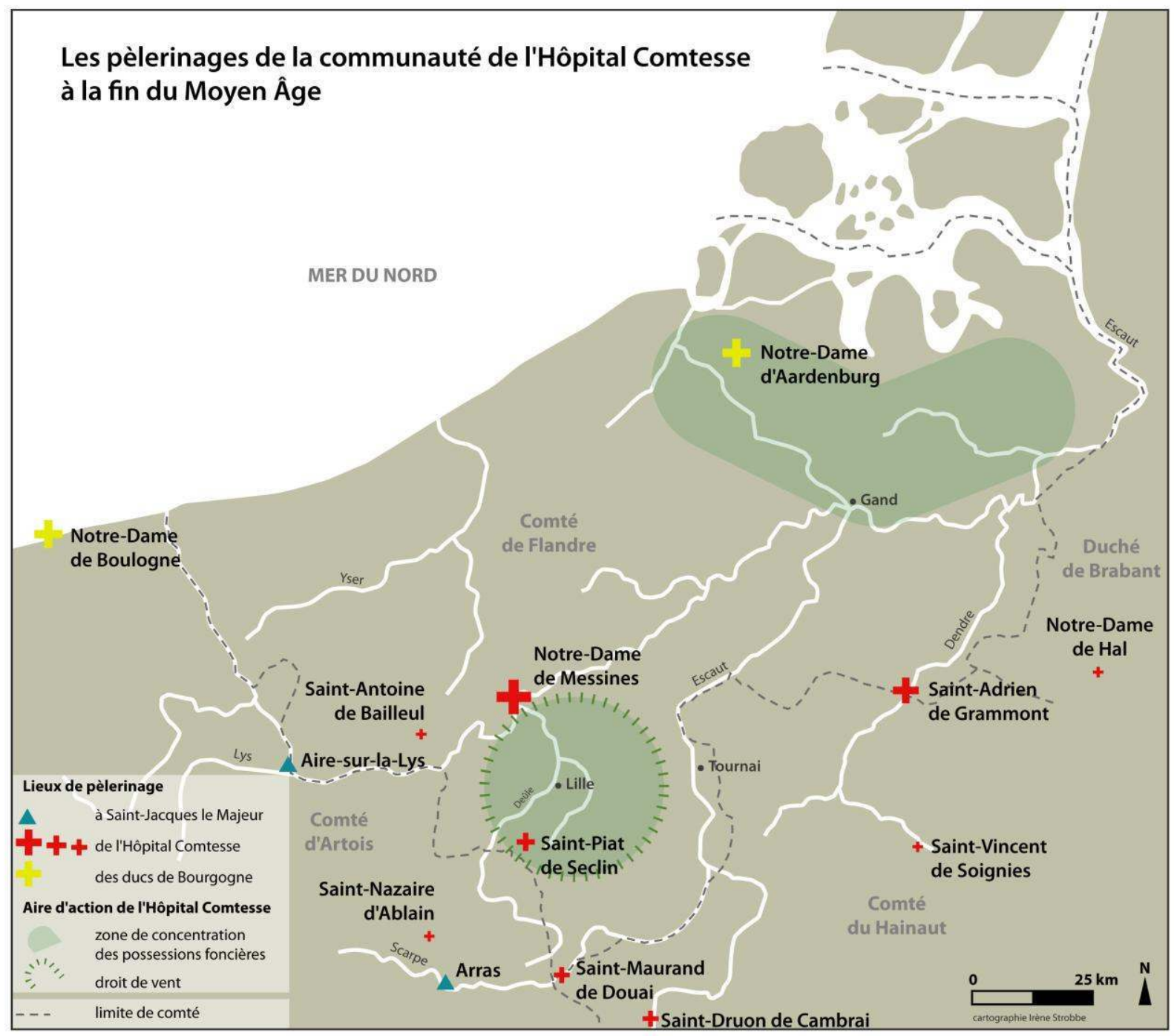

